\title{
ON THE USE OF DC MEASUREMENTS FOR ESD- RELATED PROCESS MONITORING
}

\author{
JAN MARC LUCHIES \\ MESA Research Institute, University of Twente, P.O. Box 217, 7500 AE Enschede, The Netherlands \\ FRED KUPER \\ Philips Semiconductors, MOS 3, Building FB 2.079, Gerstweg 2, 6534 AE Nijmegen, The Netherlands \\ AND \\ JAN VERWEIJ \\ MESA Research Institute, University of Twente, P.O. Box 217, 7500 AE Enschede, The Netherlands
}

\section{SUMMARY}

DC measurements and transmission line method measurements have been carried out on field oxide NMOSTs made in a $0.8 \mu \mathrm{m}$ CMOS process. Analysis shows that DC measurements can be applied for fast ESD related feedback to submicron process development.

KEY WORDS ESD Semiconductor devices Second breakdown Transmission line method TLM ESD modelling

\section{INTRODUCTION}

In process development and in production, there is a need for simple and fast evaluation of electrostatic discharge (ESD) behaviour. Automated DC measurements on the wafer would present the fastest alternative provided that there is a clear correlation between DC measurements and ESD behaviour. To assess such a correlation, DC measurements and transmission line method (TLM) measurements were carried out on field oxide NMOSTs made in a $0.8 \mu \mathrm{m}$ process technology. The DC and TLM have been compared using the rectangular box approximation for the thermal behaviour. ${ }^{1}$

In this paper, the experimental set-up and results will be presented first. Then, differences and similarities between the two types of measurements will be discussed. The methods will be compared using thermal behaviour modelling. Finally, the main parameters for ESD process monitoring will be presented.

\section{EXPERIMENTAL SET-UP}

Characterization of ESD structures is well achieved with a transmission line pulse generator. The generator produces constant current pulses with $1 \mathrm{~ns}$ risetime and pulse width dependent on the transmission line length. The high current stress pulse width was $100 \mathrm{~ns}$, which is considered closest to that of human body model ESD stress. ${ }^{2,3}$ During a pulse, the voltage and current across a test structure are measured with a fast oscilloscope (the HP54111D digital storage oscilloscope). From these data the $I-V$ characteristics of the structures can be obtained.
The devices were subjected to quasi-static DC current sweep using an HP4142B source monitor unit. A typical sweep in this investigation had a time interval of $0.1 \mathrm{~s}$ per step and a total duration of $10 \mathrm{~s}$ using a logarithmic incremental current sweep from $1 \mathrm{nA}$ to $500 \mathrm{~mA}$. Photon emission microscopy was employed on several devices to verify the current paths. ${ }^{4}$ The devices comprised field oxide NMOSTs with different gate configurations: with an aluminium gate $(\mathrm{FO}+\mathrm{Al})$, without a gate (FO) or with a polysilicon gate (FO+PS). Device layout-related parameters such as width $(W)$, length $(L)$ and the contact to LOCOS spacing $\left(R_{\mathrm{c}}\right)$ were varied (Figure 1).

Width was varied between 50 and $350 \mu \mathrm{m}$, length between 0.8 and $6 \mu \mathrm{m}$, the contact to LOCOS spacing was 2.5 to $12 \mu \mathrm{m}$. Also, rotated structures were available $\left(+45^{\circ},-45^{\circ}\right)$. The values for the collectorbase breakdown voltage and current $\left(V_{\mathrm{t} 1}, I_{\mathrm{t} 1}\right)$, the holding voltage and current $\left(V_{\mathrm{h}}, I_{\mathrm{h}}\right)$, and the second breakdown voltage and current $\left(V_{\mathrm{t} 2}, I_{\mathrm{t} 2}\right)$ were deter-

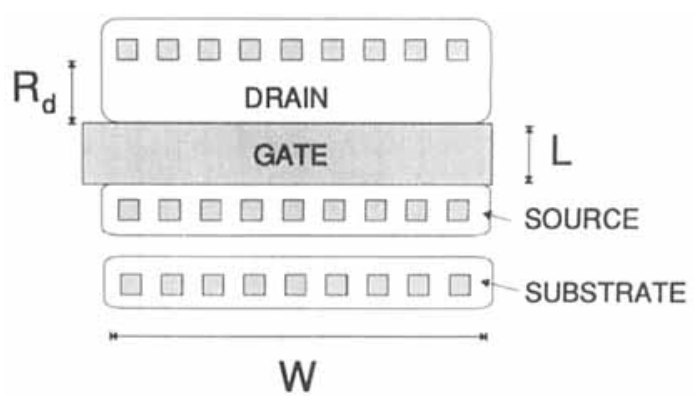

Figure 1. Device layout of the field oxide NMOST, showing the layout parameters $W, L, R_{c}$ 


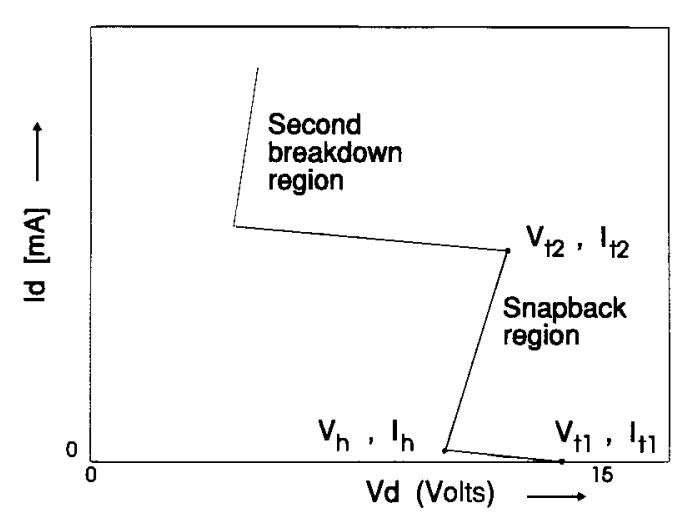

Figure 2. High-current $I / V$ characteristic

mined (Figure 2). Furthermore, the avalanching current, $V_{\mathrm{av}}$, and the differential resistance in the snapback region, $R_{\mathrm{sb}}$, were measured.

\section{EXPERIMENTAL RESULTS}

In this section, the results of the experiments will be presented. The holding voltage and the effect of the differential resistance in the snapback region on the second breakdown voltage are treated first. Then the effect of width, contact to LOCOS spacing and length are discussed.

The holding voltage and the differential resistance in the snapback region together determine the voltage shunting capabilities of the device. The holding voltages and the differential resistance in the snapback region were determined through TLM and DC measurements, where the difference between the two measurement methods was less than 5 per cent.

The dependency of the holding voltage on the device length is depicted in Figure 3 . The holding voltage is scaling with transistor length following a simple lateral $n-p-n$ transistor snapback model. The reason for this dependence can be derived from the expression for the holding voltage. ${ }^{5}$ To a first-order approximation, the holding voltage scales with the

\section{Vh vs $L$}

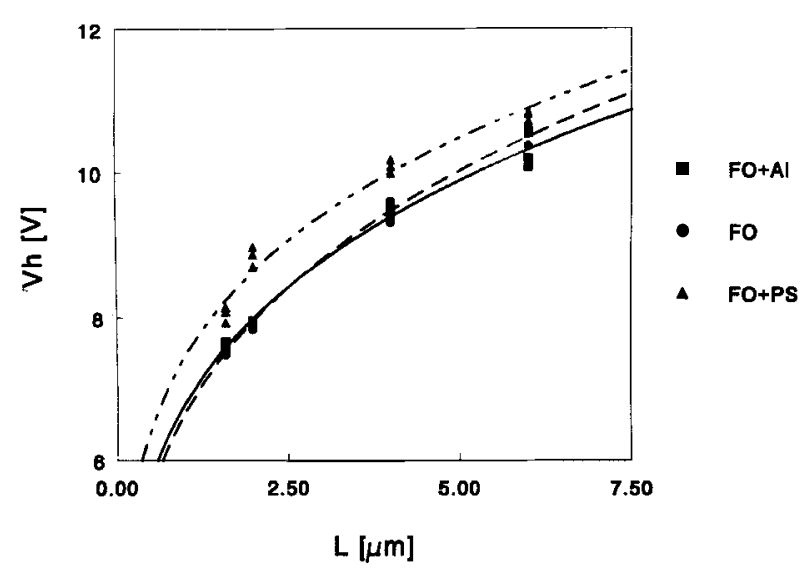

Figure 3. Holding voltages as a function of the device length

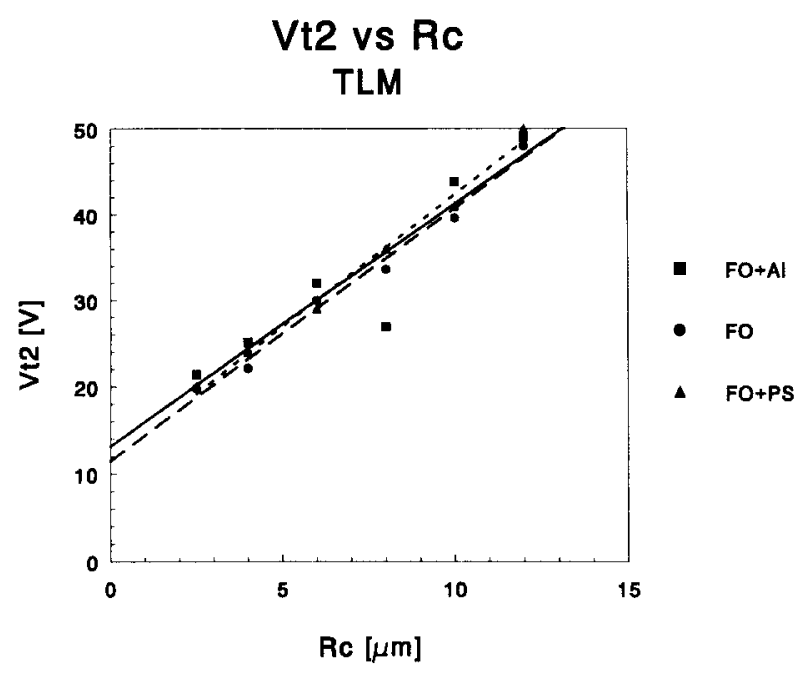

Figure 4. Effect of $R_{\mathrm{c}}$ on the second breakdown voltage

transistor length, when the exponent $\boldsymbol{n}$ in the Miller formula equals 8 .

The effect of introduction of additional series resistance, through increased $R_{\mathrm{c}}$, on the second breakdown voltage is shown in Figure 4. As expected, a simple linear relation between $R_{\mathrm{c}}$ and $V_{\mathrm{t} 2}$ is found. For high values of $R_{\mathrm{c}}$, the shunted voltage becomes high. When the device is used as a protection for an input gate, care should be taken with increasing $R_{\mathrm{c}}$.

Homogeneous current injection after snapback is decisive for high second breakdown currents. The collector-base breakdown current $I_{\mathrm{t} 1}$ can therefore already provide early information on the performance to be expected at high current levels. Deviations from normal values can give an indication of inhomogeneous current flow. Up to $175 \mu \mathrm{m}$, the collector-base breakdown current is linearly scaling with device width. For $350 \mu \mathrm{m}$ devices deviations from expected values of $I_{\mathrm{t} 1}$, obtained by extrapolating the width dependency, were observed (Figure 5 ). This already gave an indication of an orientation

\section{It1 vs $W$}

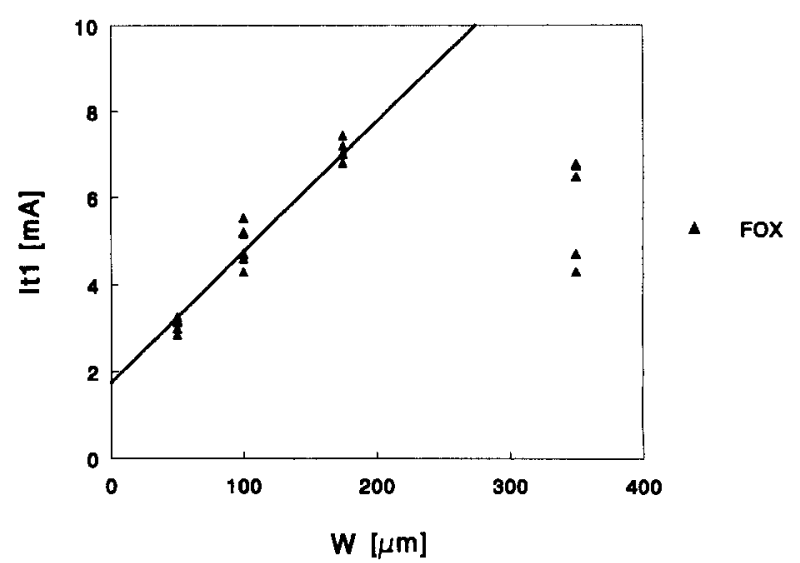

Figure 5. Collector-base breakdown currents as a function of device width 

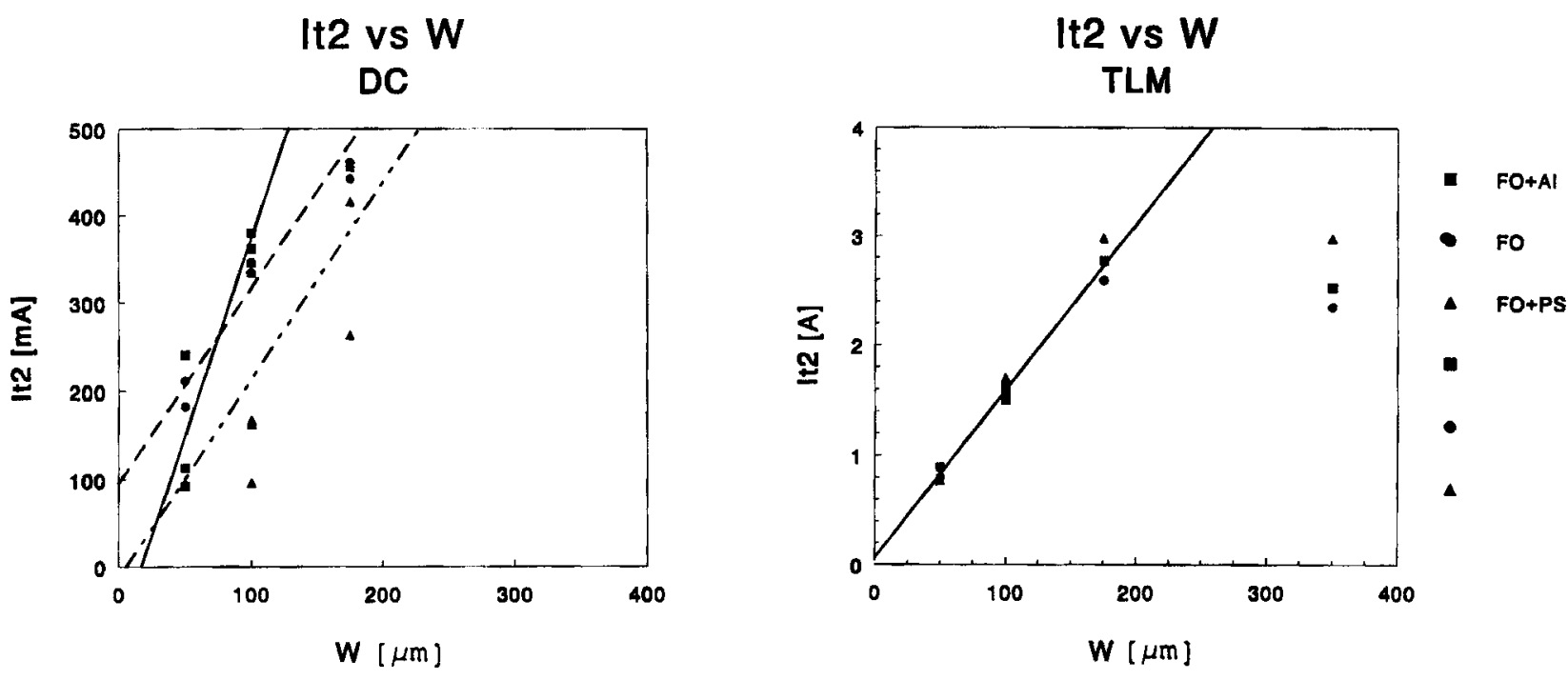

Figure 6. The effect of device width on the second breakdown current
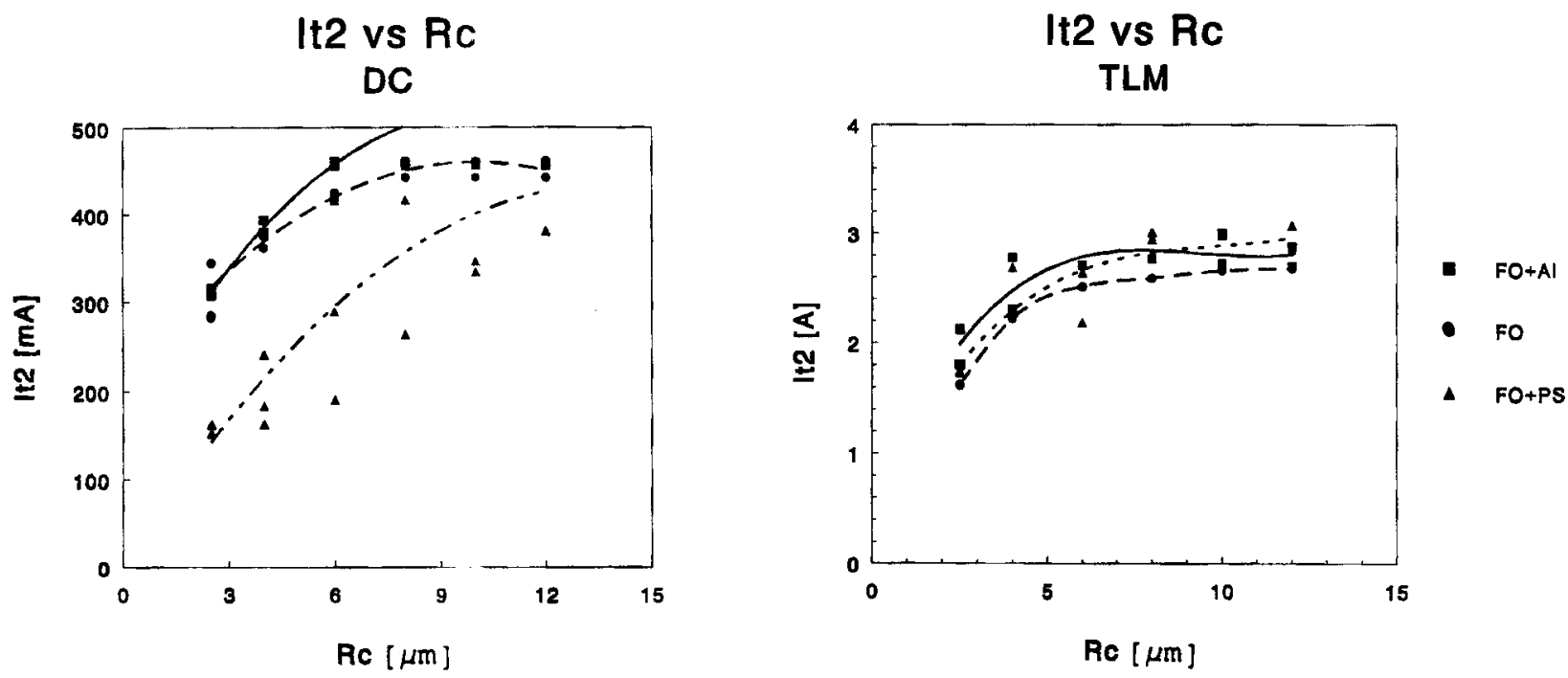

Figure 7. The effect of contact to LOCOS spacing $R_{\mathrm{c}}$ on the second breakdown current

dependency. Measurements on rotated structures confirmed this observation.

The effect of device width on the second breakdown current, $I_{\mathrm{t} 2}$, was measured both with TLM and DC, and is depicted in Figure 6. The threshold for second breakdown is linearly scaling with device width up to $175 \mu \mathrm{m}$. The $350 \mu \mathrm{m}$ device is a fingered structure $(2 \times 175 \mu \mathrm{m})$. A photon emission study showed that only one transistor was conducting after snapback. This can be attributed to the fact that the lateral doping profiles of the two transistors differ considerably due to a tilted implantation. This difference affects the collector-base breakdown voltage and current. Therefore, only one transistor will go into snapback.

The contact to LOCOS spacing is often used as a spreading resistance, which will delay the advent of hot-spot formation at the drain junction. The effect of contact to LOCOS spacing $\left(R_{\mathrm{c}}\right)$ on the second breakdown current is depicted in Figure 7. The trend in both TLM and DC measurements was found to be the same. When $R_{\mathrm{c}}$ becomes more than $7 \mu \mathrm{m}$ the second breakdown current saturates as a result of a change in failure mechanisms: from aluminium melting to silicon melting. ${ }^{6}$ For DC measurements, the field oxide transistors with polysilicon gates exhibit a larger spread in $I_{\mathrm{t} 2}$ values. This was probably due to non-uniform current flow.

Scaling of the device length is known to have no effect on the second breakdown current. Figure 8 shows the results of TLM and DC measurements. The TLM indeed do not reveal any dependency on length, whereas the DC measurements indicate length dependency. For larger gate lengths lower second breakdown currents are observed. Here, the DC measurement seems to fail in predicting the high current behaviour.

\section{DISCUSSION: COMPARISON BETWEEN DC CURRENT AND CURRENT PULSE}

During ESD events, second breakdown is the main mechanism for catastrophic damage of NMOS tran- 

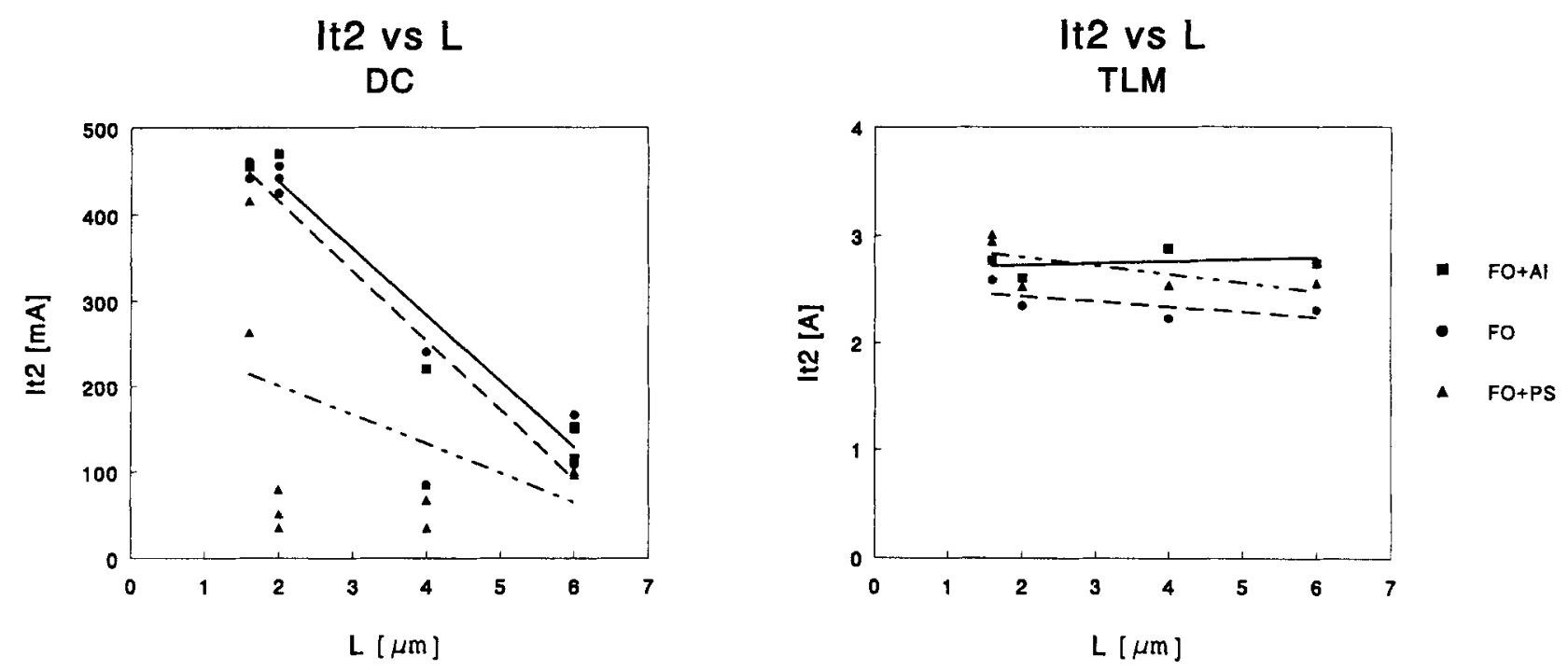

Figure 8. The length dependency of the second breakdown current measured with DC and TLM

sistors at the drain junction. Amerasekera et al. derived an expression for the ratio between the powers required to cause second breakdown under DC conditions $\left(P_{\mathrm{DC}}\right)$ and under pulsed conditions $\left(P_{\mathrm{TLM}}\right){ }^{7}$ The heat dissipated in the drain junction is modelled within a rectangular box. Comparing the expressions derived for pulsed stress and for constant current stress, and assuming that the volume in which the heat is dissipated is the same, the ratio $\left(P_{\mathrm{TLM}} / P_{\mathrm{DC}}\right)$ is given by

$$
\frac{P_{\mathrm{TLM}}}{P_{\mathrm{DC}}}=2\left[\frac{\ln (a / b)+2-c / 2 b}{\ln \left(t / t_{\mathrm{b}}\right)+2-c / b}\right]
$$

where $a, b$ and $c$ are the width, length, and diffusion depth of the rectangular box in which the dissipation is taking place. The diffusion time, $t_{\mathrm{b}}$, to reach thermal equilibrium in the lateral direction is given by $t_{\mathrm{b}}=b^{2} / 4 \pi D$, where $D$ is the thermal diffusivity.

The values of $a, b, c$ and $t_{\mathrm{b}}$ can be derived from the TLM. When the current is scaling with the device width, the current is assumed to be injected throughout the whole device width, and therefore the value of $a$ is the actual device width. That leaves only $b$ and $c$ to be determined.

The values for $b$ and $c$ were determined using a power-to-failure versus time-to-failure curve. For an average time to failure, $t$, of $50 \mathrm{~ns}$, with reasonable values for the length and depth of the rectangular box, equation (1) gave $P_{\mathrm{TLM}} / P_{\mathrm{DC}} \approx 4$. Which was comparable with the experimentally determined value $P_{\mathrm{TLM}} / P_{\mathrm{DC}}$ of 6.2 that had a spread of 0.7 for 20 measurements.

Other parameters suitable for monitoring ESD behaviour by means of DC measurements are the holding voltage $V_{\mathrm{h}}$ and the differential resistance in the snapback region. They can be applied to estimate the voltage drop at the drain, which is an important value for the determination of the protective device properties for gate oxides in input cir- cuits. Since they can be determined with reasonable accuracy with DC measurements, both can easily be used in automated measurements.

The DC measurements can effectively be applied to predict high current stress behaviour, when width or contact to LOCOS spacing are varied. The DC measurements, however, fail to predict the high current behaviour for longer device lengths, which was not observed by Amerasekera et al. This is believed to be the result of the region in which the heat is dissipated not being the same for different lengths when compared to the transistor length. For larger transistor lengths, Joule heating in the channel becomes more important. An extension of the model to include heating of adjacent areas seems appropriate. This will remain a subject for further research.

\section{CONCLUSIONS}

The correlation between high current ESD behaviour and low current quasi-static measurements shows that DC measurements can be applied to derive the ESD high current behaviour for this $0.8 \mu \mathrm{m}$ process. Using the process specific ratio $\left(P_{\mathrm{TLM}} / P_{\mathrm{DC}}\right)$, one can determine the high current ESD behaviour. Moreover, the outcome of the correlation study opens up the way for fast feedback procedures in process development. The ratio $\left(P_{\mathrm{TLM}} / P_{\mathrm{DC}}\right)$ was determined for different devices, and appears to be well in accordance with the value obtained from thermal heat equation. The main parameters for correlation of TLM and DC measurements have been identified.

\section{ACKNOWLEDGEMENTS}

These investigations in the program of the Foundation for Fundamental Research on Matter (FOM) have been supported (in part) by the Netherlands Technology Foundation (STW). 


\section{REFERENCES}

1. V. M. Dwyer, A. J. Franklin and D. S. Campbell, 'Thermal failure in semiconductor devices', Solid State Electronics, 33, (5), 553-560 (1990).

2. N. Khurana, T. Maloney and W. Yeh, 'ESD on CHMOS devices-equivalent circuits, physical models and failure mechanisms', Proc. International Reliability Physics Symposium, 1985, pp. 212-223.

3. D. G. Pierce, W. Shiley, B. D. Mulcahy, K. E. Wagner and M. Wunder, 'Electrical overstress testing of a $256 \mathrm{~K}$ UVEPROM to rectangular and double exponential pulses', Proc. 11th EOS/ESD Symposium, 1988, pp. 137-146.

4. M. Hanneman and A. Amerasekera, 'Photon emission as a tool for ESD failure localisation and as a technique for studying ESD phenomena', Proc. ESREF, 1990, pp. 77-83.

5. M. Reisch, 'On bistable behavior and open-base breakdown of bipolar transistors in the avalanche regime-modeling and applications', IEEE Trans. Electron Devices, 39, (6), 1398-1409 (1992).

6. R. N. Rountry and C. L. Hutchins, "NMOS protection circuitry', IEEE Trans. on Electron Devices, 32, (5), 910-917 (1985).

7. A. Amerasekera, L. van Roozendaal, J. Bruines and F. Kuper, 'Characterisation and modelling of second breakdown in NMOST's for the extraction of ESD-related process and design parameters', IEEE Trans. Electron Devices, 38, (9), 2161-2168, (1991).

\section{Authors' biographies:}

Jan Marc Luchies was born in Hengelo, The Netherlands in 1967. He received his M.Sc. degree in electrical engineering from the University of Twente, The Netherlands in 1991 . He is currently working towards his Ph.D. degree in electrical engineering on electrostatic discharge damage in integrated circuits at the University of Twente. His main topic is the propagation of high-current fast-transient (ESD) signals in protection devices and circuits, which is studied with the transmission line method and electrooptic sampling techniques.

Fred Kuper was born in Emmen, The Netherlands in 1959. He received his M.Sc. degree in applied physics from the University of Twente, The Netherlands in 1983. In 1987, he received the Ph.D. degree from the University of Groningen, The Netherlands, for his work on stacking faults in silicon. In 1987, he joined the IC technology division of Philips Research Laboratories, where he worked on wafer level and transistor reliability. In 1991, he joined the submicron CMOS waferfab MOS3 of Philips Semiconductors in Nijmegen, The Netherlands. His main topics there are ESD, hot carrier degradation and gate oxide reliability.

Jan Verweij was born in Benschop, The Netherlands. He studied Solid State Chemistry at the University of Utrecht. In 1967 he joined Philips Research Laboratories, Eindhoven, where he has been involved in research on hot carrier effects, oxide breakdown, high-voltage integrated circuits and non-volatile memory devices. From 1988 to 1991 he headed the Corporate Reliability Center for Integrated Circuits within Philips Components, Eindhoven.

Jan Verweij has also some teaching experience. From 1976 to 1985 he was (extraordinary) professor in Applied Physics at Groningen University; now he is (part-time again) professor in Electrical Engineering at the University of Twente. His teaching subject is 'Reliability of semiconductor devices' and he has a small research group working on modelling of electrothermal interactions, electrostatic discharge, hot carrier effects and electromigration. 